\title{
Characterization of Revenue Equivalence
}

\author{
Birgit Heydenreich ${ }^{1} \quad$ Rudolf Müller ${ }^{1} \quad$ Marc Uetz $^{1}$ \\ Rakesh Vohra ${ }^{2}$
}

May 11, 2007

\begin{abstract}
The property of an allocation rule to be implementable in dominant strategies by a unique payment scheme is called revenue equivalence. In this paper we give a characterization of revenue equivalence based on a graph theoretic interpretation of the incentive compatibility constraints. The characterization holds for any (possibly infinite) outcome space and many of the known results about revenue equivalence are immediate consequences.
\end{abstract}

\section{Introduction}

One of the most important results of auction theory is the Revenue Equivalence Theorem. Subject to certain reasonable assumptions, it concludes that a variety of different auctions generate the same expected revenue for the seller. Klemperer (1999) writes that "much of auction theory can be understood in terms of this theorem.....". Hence the long line of papers that have attempted to relax the sufficient conditions under which revenue equivalence holds. The present paper provides necessary and sufficient conditions on the underlying primitives for revenue equivalence to hold.

We consider direct revelation mechanisms for agents with multidimensional types. Such mechanisms consist of an allocation rule and a payment scheme. The allocation rule selects an outcome depending on the agents' reports about their type, whereas the

\footnotetext{
${ }^{1}$ Maastricht University, Quantitative Economics, P.O.Box 616, 6200 MD Maastricht, The Netherlands. Email:\{b.heydenreich,r.muller,m.uetz\}@ke.unimaas.nl

The first author is supported by NWO grant 2004/03545/MaGW 'Local Decisions in Decentralised Planning Environments'.

${ }^{2}$ Northwestern University, Department of Managerial Economics and Decision Sciences, Kellogg Graduate School of Management, Evanston IL 60208
} 
payment scheme assigns a payment to every agent. We focus attention on allocation rules that are implementable in dominant strategies. Hereafter we refer to such rules as implementable. In this environment we characterize the uniqueness of the relevant payment scheme in terms of conditions that are easily verified in potential applications. The property of an allocation rule to be implementable in dominant strategies by a unique payment scheme is called revenue equivalence. Our characterization of revenue equivalence is based on a graph theoretic interpretation of the incentive compatibility constraints. This graph theoretic interpretation has been used before to characterize allocation rules that are implementable in dominant strategies (see Rochet 1987, Gui, Müller, and Vohra 2004 and Saks and Yu 2005). With this interpretation, our characterizing condition for revenue equivalence almost suggests itself and the proof writes itself. The characterization holds for any (possibly infinite) outcome space. Many of the known results about revenue equivalence are immediate consequences of our characterization.

Related Work. The bulk of prior work on revenue equivalence has been devoted to identifying sufficient conditions on the type space for all allocation rules from a certain class to satisfy revenue equivalence. The papers by Green and Laffont (1977) and Holmström (1979) restrict attention to allocation rules called 'utilitarian maximizers', that is, allocation rules that maximize the sum of the valuations of all agents. Holmström, generalizing the paper by Green and Laffont, shows that when the type space is smoothly path connected then utilitarian maximizers satisfy revenue equivalence.

Myerson (1981) shows that revenue equivalence holds for every implementable rule in a setting where the type space is an interval of the real line, the outcome space is a lattice and an agents valuation for an outcome is continuous and supermodular in her type.

Krishna and Maenner (2001) derive revenue equivalence under two different hypotheses. In the first, agents' type spaces must be convex and the valuation function of an agent is a convex function of the type of the agent. Under these conditions they show that every implementable rule satisfies revenue equivalence. The second hypothesis requires the allocation rule to satisfy certain differentiability and continuity conditions and the outcome space to be a subset of the Euclidean space. Furthermore, the valuation functions must be regular Lipschitzian and monotonically increasing in all arguments.

Milgrom and Segal (2002) show that revenue equivalence is a consequence of a particular envelope theorem in a setting where the type spaces are one-dimensional and the outcome space is arbitrary. An agent's valuation function is assumed differentiable and absolutely continuous in the type of the agent and the partial derivative of the valuation function with respect to the type must satisfy a certain integrability condition. Their 
result can be applied to multi-dimensional type spaces as well. In this case the type spaces must be smoothly connected and the valuation functions must be differentiable with bounded gradient.

We know of only two papers that identify necessary as well as sufficient conditions for revenue equivalence to hold. If the outcome space is finite, Suijs (1996) characterizes type spaces and valuation functions for which utilitarian maximizers satisfy revenue equivalence. Chung and Olszewski (2007) characterize type spaces and valuation functions for which every implementable allocation rule satisfies revenue equivalence, again under the assumption of a finite outcome space. From their characterization, they derive sufficient conditions on the type spaces and valuation functions that generalize known results when the outcome space is countable or a probability distribution over a finite set of outcomes. More precisely, they can show that some of the previously known conditions can be weakened.

Our characterization result differs from prior work in an important way. We identify a condition on the type spaces, the valuation functions and the implementable allocation rule together that characterizes revenue equivalence. In other words, we prove that a particular allocation rule satisfies revenue equivalence if and only if this condition is satisfied. Our characterization result differs from the one by Chung and Olszewski (2007) in three ways. First, ours holds for general outcome spaces. Second, our result implies revenue equivalence in cases where their result does not apply. In fact, given agents' type spaces and valuation functions, several allocation rules may be implementable in dominant strategies, some of which satisfy revenue equivalence and some do not. In this case, the conditions on the type space and valuation functions from their paper obviously cannot hold. However, our characterization can be used to determine which of the allocation rules do satisfy revenue equivalence. We give a simple example in Section 7. Third, the characterization in Chung and Olszewski (2007) is a corollary of our result, in the sense that their necessary and sufficient condition is naturally related to our graph theoretic interpretation of revenue equivalence. We refer to Section 7 for details. Moreover, our characterization yields elementary and direct alternative proofs for their sufficient conditions for finite and countably infinite outcome spaces.

As in Chung and Olszewski (2007), such sufficient conditions yield a number of the earlier results as immediate consequences We list some of them below. For details, we refer to Section 7.

1. By restricting attention to countable outcome spaces we can relax in Holmström (1979) the smooth connectedness condition on the type space to (topological) con- 
nectedness. In addition, our sufficient condition applies to any allocation that can be implemented in dominant strategies rather than just utilitarian maximizers.

2. The sufficient condition that Suijs (1996) derives from his characterization follows as a special case.

3. The sufficient condition of Krishna and Maenner (2001) under their first hypothesis when the outcome space is countable follows as a special case.

4. The sufficient condition of Milgrom and Segal (2002) when the outcome space is countable follows as a special case.

The remainder of the paper is organized as follows. In Section 2 we introduce notation and basic definitions. In Section 3, we recall and prove some graph theoretic results. These are applied in Section 4 to derive our main result, and in Sections 5 and 6 to provide sufficient conditions for finite and countably infinite outcome spaces, respectively. In Section 7 we show how the characterization from Chung and Olszewski (2007) can be obtained as a consequence of ours and give an example to illustrate the differences between our characterization and theirs. We conclude with extensions of our characterization result to other notions of incentive compatibility in Section 8.

\section{Setting and Basic Concepts}

Denote by $\{1, \ldots, n\}$ the set of agents and let $A$ be the set of possible outcomes. Outcome space $A$ is allowed to have infinitely many, even uncountably many, elements. By $t_{i}$, we denote the type of agent $i \in\{1, \ldots, n\}$. Let $T_{i}$ be the type space of agent $i$. Type spaces $T_{i}$ can be arbitrary sets. Agent $i$ 's preferences over outcomes are modeled by the valuation function $v_{i}: A \times T_{i} \rightarrow \mathbb{R}$, where $v_{i}\left(a, t_{i}\right)$ is the valuation of agent $i$ for outcome $a$ when he has type $t_{i}$.

A mechanism $(f, \pi)$ consists of an allocation rule $f: \prod_{i=1}^{n} T_{i} \rightarrow A$ and a payment scheme $\pi: \prod_{i=1}^{n} T_{i} \rightarrow \mathbb{R}^{n}$. In a direct revelation mechanism, the allocation rule chooses for a vector $t$ of aggregate type reports of all agents an outcome $f(t)$, whereas the payment scheme assigns a payment $\pi_{i}(t)$ to each agent $i$. Let the vector $\left(t_{i}, t_{-i}\right)$ denote the aggregate type report vector when $i$ reports $t_{i}$ and the other agents' reports are represented by $t_{-i}$. We assume quasi-linear utilities, that is, the utility of agent $i$ when the aggregate report vector is $\left(t_{i}, t_{-i}\right)$ is $v_{i}\left(f\left(t_{i}, t_{-i}\right), t_{i}\right)-\pi_{i}\left(t_{i}, t_{-i}\right)$.

In a truthful mechanism, truth telling is a (weakly) dominant strategy for every agent. 
Definition 1 (dominant strategy incentive compatible) A direct revelation mechanism $(f, \pi)$ is called dominant strategy incentive compatible if for every agent $i$, every type $t_{i} \in T_{i}$, all aggregate type vectors $t_{-i}$ that the other agents could report and every type $s_{i} \in T_{i}$ that $i$ could report instead of $t_{i}$ :

$$
v_{i}\left(f\left(t_{i}, t_{-i}\right), t_{i}\right)-\pi_{i}\left(t_{i}, t_{-i}\right) \geq v_{i}\left(f\left(s_{i}, t_{-i}\right), t_{i}\right)-\pi_{i}\left(s_{i}, t_{-i}\right) .
$$

If for allocation rule $f$ there exists a payment scheme $\pi$ such that $(f, \pi)$ is a truthful mechanism, then $f$ is called implementable in dominant strategies, short implementable.

With the exception of Section 8, we assume that the allocation rule is implementable in dominant strategies and we study the uniqueness of the corresponding payment scheme. We refer to the latter as revenue equivalence.

Definition 2 (Revenue Equivalence) An allocation rule $f$ that is implementable in dominant strategies satisfies the revenue equivalence property if for any two dominant strategy incentive compatible mechanisms $(f, \pi)$ and $\left(f, \pi^{\prime}\right)$ and any agent $i$ there exists a function $h_{i}$ that only depends on the reported types of the other agents $t_{-i}$ such that

$$
\forall t_{i} \in T_{i}: \pi_{i}\left(t_{i}, t_{-i}\right)=\pi_{i}^{\prime}\left(t_{i}, t_{-i}\right)+h_{i}\left(t_{-i}\right)
$$

\section{Unique Node Potentials in Directed Graphs}

In this section, we prove two theorems about node potentials in directed graphs. The first theorem yields a necessary and sufficient condition for a graph to have a node potential that is uniquely defined up to a constant. The second theorem provides another sufficient condition for uniqueness of the node potential up to a constant. In the following sections we will make use of these results to obtain necessary and sufficient conditions for revenue equivalence to hold.

Let $G=(V, E)$ be a directed graph with node set $V$ and arc set $E$. $V$ is allowed to be infinite. By $\ell_{a b}$ we denote the (finite) length of the arc $(a, b)$ from node $a$ to node $b$. A path from node $a$ to node $b$ in $G$, or short $(a, b)$-path, is defined as $P=\left(a=a_{0}, a_{1}, \ldots, a_{k}=b\right)$ such that $\left(a_{i-1}, a_{i}\right) \in E$ for $i=1, \ldots, k$. Denote by length $(P)$ the length of this path. A cycle is a path with $a=b$. For any $a$, we regard the path from $a$ to $a$ without any edges as $(a, a)$-path as well and define its length as 0 . We assume that $G$ is strongly connected, that is, between any two nodes $a, b \in V$, there exists an $(a, b)$-path and a $(b, a)$-path. Define $\mathcal{P}(a, b)$ to be the set of all $(a, b)$-paths. 
Definition 3 (Node Potential) A node potential $p$ is a function $p: V \rightarrow \mathbb{R}$ such that for all arcs $(x, y) \in E, p(y) \leq p(x)+\ell_{x y}$.

Lemma 1 A graph $G$ has a node potential if and only if it has no cycle of negative length.

Proof. For finite node sets $V$, this result is well known. In graphs without negative cycles it is possible to compute shortest paths from a fixed node $a$. The lengths $\operatorname{dist}_{G}(a, \cdot)$ of these paths define a node potential. On the other hand, adding up the inequalities $p(y)-p(x) \leq \ell_{x y}$ for all edges $(x, y)$ on a cycle proves that the cycle has nonnegative length.

The proof for the finite case can be easily extended to the non-finite case, see for example Rochet (1987) and Müller, Perea, and Wolf (2007).

If $G$ does not contain a negative cycle, we say that it satisfies the nonnegative cycle property. In the following assume that $G$ satisfies the nonnegative cycle property. Let

$$
\operatorname{dist}_{G}(a, b)=\inf _{P \in \mathcal{P}(a, b)} \text { length }(P) .
$$

If $V$ is a finite set, then $\operatorname{dist}_{G}(a, b)$ simply equals the length of a shortest path from $a$ to $b$ in $G$. For infinite $V$, such a shortest path may not exist. Nevertheless, $\operatorname{dist}_{G}(a, b)$ is finite, since we assume that $G$ does not have any negative cycle. In fact, fix some $(b, a)$-path $P_{b a}$, then length $(P) \geq-$ length $\left(P_{b a}\right)$ holds for every $(a, b)$-path $P$ and the infimum is finite. The next theorem is concerned with the uniqueness of a node potential in a graph without negative cycles.

Theorem 1 Let $G=(V, E)$ be a strongly connected directed graph that satisfies the nonnegative cycle property. Then the following statements are equivalent.

1. Any two node potentials in $G$ differ only by a constant.

2. For all $a, b \in V$, $\operatorname{dist}_{G}(a, b)+\operatorname{dist}_{G}(b, a)=0$.

Proof. $[1 \Rightarrow 2]$ The function $\operatorname{dist}_{G}(a, \cdot): V \rightarrow \mathbb{R}$ assigns to every node $x \in V$ the infimum over the lengths of all $(a, x)$-paths. Clearly, $\operatorname{dist}_{G}(a, x) \leq \operatorname{dist}_{G}(a, y)+\ell_{y x}$ for all $(y, x) \in E$ and therefore $\operatorname{dist}_{G}(a, \cdot)$ is a node potential in $G$. Similarly, $\operatorname{dist}_{G}(b, \cdot)$ is a node potential. As any two node potentials differ only by a constant, we have that $\operatorname{dist}_{G}(a, \cdot)-\operatorname{dist}_{G}(b, \cdot)$ is a constant function. Especially, for $a$ and $b$ we get that $\operatorname{dist}_{G}(a, a)-\operatorname{dist}_{G}(b, a)=\operatorname{dist}_{G}(a, b)-\operatorname{dist}_{G}(b, b)$. Clearly, $\operatorname{dist}_{G}(a, a)=\operatorname{dist}_{G}(b, b)=0$ and hence $\operatorname{dist}_{G}(a, b)+\operatorname{dist}_{G}(b, a)=0$. 
$[2 \Rightarrow 1]$ Let $a, b \in V$. Let $P_{a b}$ be an $(a, b)$-path with nodes $a=a_{0}, a_{1}, \ldots, a_{k}=b$. For any node potential $p$ we have that

$$
\begin{aligned}
p\left(a_{1}\right)-p(a) & \leq \ell_{a a_{1}} \\
p\left(a_{2}\right)-p\left(a_{1}\right) & \leq \ell_{a_{1} a_{2}} \\
& \vdots \\
p(b)-p\left(a_{k-1}\right) & \leq \ell_{a_{k-1} b}
\end{aligned}
$$

and consequently $p(b)-p(a) \leq$ length $\left(P_{a b}\right)$. Therefore,

$$
p(b)-p(a) \leq \inf _{P \in \mathcal{P}(a, b)} \text { length }(P)=\operatorname{dist}_{G}(a, b)
$$

Similarly, $p(a)-p(b) \leq \operatorname{dist}_{G}(b, a)$. Therefore, $-\operatorname{dist}_{G}(b, a) \leq p(b)-p(a) \leq \operatorname{dist}_{G}(a, b)$. Since $\operatorname{dist}_{G}(a, b)=-\operatorname{dist}_{G}(b, a), p(b)-p(a)=\operatorname{dist}_{G}(a, b)$ for any node potential $p$. Hence, any potential is completely defined, once $p(a)$ has been chosen for some outcome $a$. Thus, any two node potentials can only differ by a constant.

Next, we define a property of the graph $G$ that is sufficient (though not necessary) for uniqueness of node potentials up to a constant.

Definition 4 (Two-Cycle Connected) A graph with node set $V$ and arc lengths $\ell$ is called two-cycle connected if for every partition $V_{1} \cup V_{2}=V, V_{1} \cap V_{2}=\emptyset, V_{1}, V_{2} \neq \emptyset$, there are $a_{1} \in V_{1}$ and $a_{2} \in V_{2}$ with $\ell_{a_{1} a_{2}}+\ell_{a_{2} a_{1}}=0$.

Theorem 2 Let $G$ be a directed graph that satisfies the nonnegative cycle property. If $G$ is two-cycle connected then its node potential is uniquely defined up to a constant.

Proof. First, we show that if $G$ is two-cycle connected, then any two nodes $a, b \in V$ are connected in $G$ by a finite path with nodes $a=a_{0}, a_{1}, \ldots, a_{k}=b$ such that $\ell_{a_{i} a_{i+1}}+\ell_{a_{i+1} a_{i}}=$ 0 for $i=0, \ldots, k-1$. Call such a path a zero-path. Suppose to the contrary, that there is a node $a \in V$ that is not connected to all nodes in $G$ by a zero-path. Define $V_{1}$ to be the set containing all nodes $b$ that can be reached from $a$ by a zero-path. Let $V_{2}=V \backslash V_{1}$. By assumption $V_{2} \neq \emptyset$. Then, as $G$ is two-cycle connected, there is an $a_{1} \in V_{1}$ and $a_{2} \in V_{2}$ with $\ell_{a_{1} a_{2}}+\ell_{a_{2} a_{1}}=0$ contradicting the assumption that $a_{2} \in V_{2}$.

Consider $a, b \in V$ and a zero-path $P_{a b}=\left(a_{0}, a_{1}, \ldots, a_{k}\right)$. Then $P_{a b}$ together with the $(b, a)$-path $P_{b a}=\left(a_{k}, \ldots, a_{1}, a_{0}\right)$ form a cycle of length 0 . Note, that between any two nodes $c, d$ on a cycle of length 0 , the path from $c$ to $d$ on the cycle must be a shortest path, as otherwise, we could construct a negative cycle by substituting this path by a 
shorter one. Therefore, $\operatorname{dist}_{G}(a, b)+\operatorname{dist}_{G}(b, a)=\operatorname{length}\left(P_{a b}\right)+\operatorname{length}\left(P_{b a}\right)=0$. Hence, any two node potentials in $G$ differ only by a constant due to Theorem 1.

To see that two-cycle connectedness is not necessary for the uniqueness of the node potential, consider the following example.

Example 1 Consider graph $G$ in Figure 1. The graph satisfies the nonnegative cycle property, and for every two nodes $u, v \in V$, $\operatorname{dist}_{G}(u, v)+\operatorname{dist}_{G}(v, u)=0$. Hence, the node potential is uniquely defined up to a constant according to Theorem 1. Notice, however, that $G$ is not two-cycle connected, as the partition $(\{a, c\},\{b\})$ violates the condition of Definition 4 .

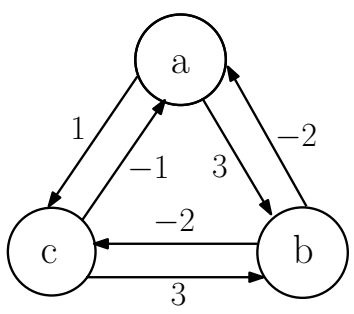

Figure 1: Example

\section{Characterization of Revenue Equivalence}

We prove a necessary and sufficient condition for revenue equivalence with the aid of a graph theoretic interpretation used by Rochet (1987), Gui et al. (2004) and Saks and $\mathrm{Yu}(2005)$ to characterize implementable allocation rules. We also adopt some of their notation.

Fix agent $i$ and let the reports of the other agents $t_{-i}$ be fixed as well. For simplicity of notation we write $T$ and $v$ instead of $T_{i}$ and $v_{i}$. Similarly, for any mechanism $(f, \pi)$, we regard $f$ and $\pi$ as functions of $i$ 's type alone, i.e. $f: T \rightarrow A$ and $\pi: T \rightarrow \mathbb{R}$. If $(f, \pi)$ is dominant strategy incentive compatible, it is easy to see that for any pair of types $s, t \in T$ such that $f(t)=f(s)=a$ for some $a \in A$, the payments must be equal, i.e. $\pi(t)=\pi(s)=: \pi_{a}$. Hence, the payment of agent $i$ is completely defined if the numbers $\pi_{a}$ are defined for all outcomes $a \in A$ such that $f^{-1}(a)$ is nonempty. Therefore, we may without loss of generality restrict attention to the case where $f$ is onto. For an allocation rule $f$, let us define the complete directed and possibly infinite allocation graph $G_{f}$ with 
node set $A^{3}$. Between any two nodes $a, b \in A$, there is a directed arc with length ${ }^{4}$

$$
\ell_{a b}=\inf _{t \in f^{-1}(b)}(v(b, t)-v(a, t))
$$

As $G_{f}$ is complete, it is also strongly connected.

Observation 1 Let $f$ be an allocation rule. Payment schemes $\pi$ such that $(f, \pi)$ is a dominant strategy incentive compatible mechanism, correspond to node potentials in each of the allocation graphs $G_{f}$ that are obtained from a combination of an agent and a report vector of the other agents.

Proof. Assume $f$ is implementable. Fix agent $i$ and the reports $t_{-i}$ of the other agents. Regard the corresponding allocation graph $G_{f}$. As already mentioned above, for any pair of types $s, t \in T$ such that $f(t)=f(s)=a$ for some $a \in A$, the payments must be equal, i.e. $\pi(t)=\pi(s)=\pi_{a}$. Therefore, $\pi$ is indeed a function assigning a real number to every node in the graph. Incentive compatibility implies for any two outcomes $a, b \in A$ and all $t \in f^{-1}(b)$ that $v(b, t)-\pi_{b} \geq v(a, t)-\pi_{a}$, hence, $\pi_{b} \leq \pi_{a}+\ell_{a b}$. The latter is the definition of a node potential in $G_{f}$.

For the other direction, define the payment $\pi$ for agent $i$ as follows. For any report vector of the other agents $t_{-i}$, consider the corresponding allocation graph $G_{f}$. At aggregate report vector $\left(t_{i}, t_{-i}\right)$ with outcome $a=f\left(t_{i}, t_{-i}\right)$, let the payment be equal to a node potential $\pi_{a}$ in $G_{f}$. Incentive compatibility now follows from the fact that $\pi$ is a node potential, similarly to the above.

Clearly, the allocation graphs can be defined for any allocation rule such that all arc lengths are finite. Observation 1 together with Theorem 1 therefore also yields a characterization of allocation rules that are implementable in dominant strategies.

Observation 2 The allocation rule $f$ is implementable in dominant strategies if and only if all allocation graphs $G_{f}$ obtained from a combination of an agent and a report vector of the other agents satisfy the nonnegative cycle property.

From the observations above it follows that for any allocation rule $f$ that is implementable in dominant strategies, there exist node potentials in all allocation graphs $G_{f}$.

\footnotetext{
${ }^{3}$ Clearly, the allocation graph depends on the agent $i$ and reports $t_{-i}$ of the other agents. In order to keep notation simple, we will suppress this dependence on $i$ and $t_{-i}$ and will simply write $G_{f}$.

${ }^{4}$ We assume that arc length are strictly larger than $-\infty$. For allocation rules implementable in dominant strategies this is no restriction, as the incentive compatibility constraints imply finiteness of the arc lengths.
} 
Furthermore, $f$ satisfies revenue equivalence if and only if in all allocation graphs $G_{f}$ the node potential is uniquely defined up to a constant. Combining this with Theorem 1 yields our main theorem.

Theorem 3 (Characterization of Revenue Equivalence) Let $f$ be an allocation rule that is implementable in dominant strategies. Then $f$ satisfies revenue equivalence if and only if in all allocation graphs $G_{f}$ obtained from a combination of an agent and a report vector of the other agents, dist $_{G_{f}}(a, b)+\operatorname{dist}_{G_{f}}(b, a)=0$ for all $a, b \in A$.

To conclude this section, we give an illustrating example.

Example 2 Consider one agent with type space $T=\{x, y, z\}$ and let the outcome space be $A=\{a, b, c\}$. The valuations are given by the following table.

\begin{tabular}{|l|lll|}
\hline & $\mathrm{a}$ & $\mathrm{b}$ & $\mathrm{c}$ \\
\hline$x$ & 2 & 4 & 3 \\
$y$ & 1 & 4 & 1 \\
$z$ & 2 & 5 & 3 \\
\hline
\end{tabular}

Consider allocation rule $f$ with $f(x)=a, f(y)=b, f(z)=c$. Then the allocation graph is the graph $G_{f}$ pictured in Figure 1 of Section 3. The allocation rule $f$ is implementable in dominant strategies, as all cycles in the graph are non-negative. As $G_{f}$ satisfies the distance condition from Theorem 3, revenue equivalence holds.

\section{Application to Finite Outcome Spaces}

In this section, we prove revenue equivalence for finite outcome spaces when type spaces and valuation functions satisfy very weak assumptions. From now on, we assume that type spaces are subsets of a Euclidian space, i.e. $T_{i} \subseteq \mathbb{R}^{k_{i}}$ for every agent $i$. Recall that a subset $T$ of a topological space is connected if it cannot be covered non-trivially by the disjoint union of two open sets. That is, there exist no open sets $T_{1}, T_{2}$ with $T \subseteq T_{1} \cup T_{2}$, $T_{1} \cap T_{2}=\emptyset, T \cap T_{1} \neq \emptyset$ and $T \cap T_{2} \neq \emptyset$. We prove the following.

Theorem 4 Let $A$ be a finite outcome space. Let each agent $i \in\{1, \ldots, n\}$ have types from the (topologically) connected type space $T_{i} \subseteq \mathbb{R}^{k_{i}}$. Let each agent's valuation function $v_{i}(a, \cdot)$ be a continuous function in the type of the agent for every $a \in A$.

Then, every allocation rule $f: \Pi_{i=1}^{n} T_{i} \rightarrow A$ that is implementable in dominant strategies satisfies revenue equivalence. 
Independently Chung and Olszewski (2007) show how to derive Theorem 4 from their characterization of revenue equivalence. We refer the reader to Section 7 for further details. In order to prove Theorem 4, we need the following fact from topology that can be found e.g. in Munkres (2000).

Fact 1 Let $T \subseteq \mathbb{R}^{k}$ be a connected set. Then any partition of $T$ into subsets $T_{1}, T_{2} \neq \emptyset$, $T_{1} \cup T_{2}=T, T_{1} \cap T_{2}=\emptyset$ satisfies $\bar{T}_{1} \cap \bar{T}_{2} \neq \emptyset$, where $\bar{T}_{i}$ is the closure of $T_{i}$ in $T$.

Now, we are able to prove the theorem.

Proof (Theorem 4). Consider a single agent with type space $T$ and valuation function $v$. Regard $f$ as a function on $T$ as before. Let $A_{1} \cup A_{2}=A, A_{1} \cap A_{2}=\emptyset, A_{1}, A_{2} \neq \emptyset$ be a partition of $A$. Then, $T=f^{-1}\left(A_{1}\right) \cup f^{-1}\left(A_{2}\right), f^{-1}\left(A_{1}\right) \cap f^{-1}\left(A_{2}\right)=\emptyset$ is a partition of $T$ and $f^{-1}\left(A_{1}\right), f^{-1}\left(A_{2}\right) \neq \emptyset$, since $f$ is onto. According to the fact above, there exists $t \in \overline{f^{-1}\left(A_{1}\right)} \cap \overline{f^{-1}\left(A_{2}\right)}$. Hence, there are sequences $\left(t_{1}^{n}\right) \subseteq f^{-1}\left(A_{1}\right)$ and $\left(t_{2}^{n}\right) \subseteq f^{-1}\left(A_{2}\right)$ with $\lim _{n \rightarrow \infty} t_{1}^{n}=\lim _{n \rightarrow \infty} t_{2}^{n}=t$. As $A$ is finite, there must be $a_{1} \in A_{1}$ and $a_{2} \in A_{2}$ and subsequences $\left(t_{1}^{n_{k}}\right) \subseteq\left(t_{1}^{n}\right)$ and $\left(t_{2}^{n_{m}}\right) \subseteq\left(t_{2}^{n}\right)$ with $f\left(t_{1}^{n_{k}}\right)=a_{1}$ for all $k$ and $f\left(t_{2}^{n_{m}}\right)=a_{2}$ for all $m$. Since $v$ is continuous in the type,

$$
\begin{aligned}
0 & =v\left(a_{2}, t\right)-v\left(a_{1}, t\right)+v\left(a_{1}, t\right)-v\left(a_{2}, t\right) \\
& =\lim _{n \rightarrow \infty}\left(v\left(a_{2}, t_{2}^{n_{m}}\right)-v\left(a_{1}, t_{2}^{n_{m}}\right)+v\left(a_{1}, t_{1}^{n_{k}}\right)-v\left(a_{2}, t_{1}^{n_{k}}\right)\right) .
\end{aligned}
$$

According to the definition of the arc length in $G_{f}$, the latter can be bounded from below as follows.

$$
\lim _{n \rightarrow \infty}\left(v\left(a_{2}, t_{2}^{n_{m}}\right)-v\left(a_{1}, t_{2}^{n_{m}}\right)+v\left(a_{1}, t_{1}^{n_{k}}\right)-v\left(a_{2}, t_{1}^{n_{k}}\right)\right) \geq \ell_{a_{1} a_{2}}+\ell_{a_{2} a_{1}} \geq 0 .
$$

The last inequality is true, since $G_{f}$ has no negative cycles. Hence, all inequalities are equalities and $\ell_{a_{1} a_{2}}+\ell_{a_{2} a_{1}}=0$. Consequently, $G_{f}$ is two-cycle connected. The claim follows from Theorem 2 and Observation 1.

Notice that we cannot omit the continuity assumption, as the following example demonstrates.

Example 3 Let there be one agent with type $t \in[0,1]$ and two outcomes $A=\{a, b\}$. Let the agent's valuation be $v(a, t)=1$, if $t<1 / 2$ and $v(a, t)=0$, if $t \geq 1 / 2$. Let $v(b, t)=1 / 2$ for all $t$. That is, $v(a, \cdot)$ is discontinuous at $t=1 / 2$. Let the allocation rule be the efficient one, i.e., $f(t)=a$ for $t<1 / 2$ and $f(t)=b$ otherwise. Then dominant strategy incentive 
compatibility is equivalent to $1-\pi_{a} \geq 1 / 2-\pi_{b}$ and $1 / 2-\pi_{b} \geq-\pi_{a}$, which is satisfied whenever $\left|\pi_{a}-\pi_{b}\right| \leq 1 / 2$. For instance, $\pi_{a}=\pi_{b}=0$ or $\pi_{a}^{\prime}=1 / 2, \pi_{b}^{\prime}=0$ are two payment schemes that make $f$ truthful, but $\pi$ and $\pi^{\prime}$ do not differ by a constant.

If the type space is not connected, there are examples of the same flavor as Example 3, where the payment scheme is not unique. However, even if the valuation function is not continuous everywhere, sufficient conditions for revenue equivalence can be proven using Theorem 2. Indeed, if the valuation functions are continuous at particular type vectors as they are constructed in the proof, two-cycle connectedness of the graphs $G_{f}$ follows. Also, if $T$ is path connected and between any two types there exists a continuous path such that the valuation functions are continuous along this path, we can make use of Theorem 2.

\section{Countably Infinite Outcome Spaces}

Inspired by Chung and Olszewski (2007), we investigate the case of countably infinite outcome spaces in this section. Again, the graph theoretic results from Section 3 yield an elementary proof for revenue equivalence under weak assumptions, which we consider to be simpler than the proof by Chung and Olszewski for the same result.

Let $A$ be a countably infinite outcome set. For the moment, we identify the type space of agent $i$ with the set of infinite vectors that have a component for every outcome. That is, $T_{i} \subseteq \mathbb{R}^{A}$ and $t_{a}=v_{i}(a, t)$. Our aim is to prove a result similar to Theorem 4 . We equip $\mathbb{R}^{A}$ with a topology that is defined by means of $\varepsilon$-balls. Let $B_{\varepsilon}(t)=\{s \in$ $\mathbb{R}^{A}$ s.t. $\left.\sup _{a \in A}\left|t_{a}-s_{a}\right|<\varepsilon\right\}$ be the $\varepsilon$-ball around $t$ in $\mathbb{R}^{A}$. Define an open set in $\mathbb{R}^{A}$ as a set $S \subseteq \mathbb{R}^{A}$ such that $t \in S$ implies that $B_{\varepsilon}(t) \subseteq S$ for some $\varepsilon>0$. It is straightforward to check that this indeed defines a topology on $\mathbb{R}^{A}$. We prove the following theorem that can also be found in Chung and Olszewski (2007).

Theorem 5 Let $A$ be a countable outcome space. Let the type space $T_{i} \subseteq \mathbb{R}^{A}$ for every agent $i$ be (topologically) connected with respect to the above defined topology.

Then, every allocation rule $f: \prod_{i=1}^{n} T_{i} \rightarrow A$ that is implementable in dominant strategies satisfies revenue equivalence.

Note that Theorem 5 generalizes Theorem 4. While the proof of Theorem 4 follows easily from Theorem 2, this approach fails in the infinite case, and in fact, our proof of Theorem 5 is based on Theorem 1 rather than 2. That is why we gave the case of finite $A$ an independent treatment in the previous section. 
Proof (Theorem 5). Let $f$ be an allocation rule implementable in dominant strategies which does not satisfy revenue equivalence. According to Theorem 3, there is an agent $i$ and a report vector of the other agents $t_{-i}$ with corresponding allocation graph $G_{f}$ and $a^{*}, b^{*} \in A$ such that $\operatorname{dist}_{G_{f}}\left(a^{*}, b^{*}\right)+\operatorname{dist}_{G_{f}}\left(b^{*}, a^{*}\right)>0$. Let $T$ be the type space of $i$ and regard $f$ as a function on $T$ as before. As $A$ is countable, the set $\left\{\operatorname{dist}_{G_{f}}\left(a^{*}, x\right)+\right.$ $\left.\operatorname{dist}_{G_{f}}\left(x, a^{*}\right) \mid x \in A\right\}$ contains only countably many values ${ }^{5}$. Hence, there exists a $\delta>0$ such that the sets $A_{1}=\left\{x \mid \operatorname{dist}_{G_{f}}\left(a^{*}, x\right)+\operatorname{dist}_{G_{f}}\left(x, a^{*}\right)<\delta\right\}$ and $A_{2}=\left\{x \mid \operatorname{dist}_{G_{f}}\left(a^{*}, x\right)+\right.$ $\left.\operatorname{dist}_{G_{f}}\left(x, a^{*}\right)>\delta\right\}$ are both non-empty and together yield a partition of $A$. Then, the sets $T_{1}=f^{-1}\left(A_{1}\right)$ and $T_{2}=f^{-1}\left(A_{2}\right)$ are non-empty and yield a partition of the type space $T$. $T_{1}$ is a proper subset of $T$. We will show that $T_{1}$ is open and closed, implying that $T$ is not connected.

$T_{1}$ is open: Let $t \in T_{1}$. Let $f(t)=x \in A_{1}$. Then, $\operatorname{dist}_{G_{f}}\left(a^{*}, x\right)+\operatorname{dist}_{G_{f}}\left(x, a^{*}\right)=\delta-\varepsilon$ for some $\varepsilon=\varepsilon(x)>0$. Let $s \in B_{\varepsilon / 2}(t)$ and $y=f(s)$. Then the following is true:

$$
\begin{aligned}
\operatorname{dist}_{G_{f}}\left(a^{*}, y\right)+\operatorname{dist}_{G_{f}}\left(y, a^{*}\right) & \leq \operatorname{dist}_{G_{f}}\left(a^{*}, x\right)+\ell_{x y}+\ell_{y x}+\operatorname{dist}_{G_{f}}\left(x, a^{*}\right) \\
& \leq \operatorname{dist}_{G_{f}}\left(a^{*}, x\right)+\operatorname{dist}_{G_{f}}\left(x, a^{*}\right)+s_{y}-s_{x}+t_{x}-t_{y} \\
& \leq \operatorname{dist}_{G_{f}}\left(a^{*}, x\right)+\operatorname{dist}_{G_{f}}\left(x, a^{*}\right)+\left|s_{y}-t_{y}\right|+\left|t_{x}-s_{x}\right| \\
& <\delta-\varepsilon+\frac{\varepsilon}{2}+\frac{\varepsilon}{2} \\
& =\delta
\end{aligned}
$$

Hence $\operatorname{dist}_{G_{f}}\left(a^{*}, y\right)+\operatorname{dist}_{G_{f}}\left(y, a^{*}\right)<\delta$. Thus $y \in A_{1}$ and $s \in T_{1}$. Consequently, $B_{\varepsilon / 2}(t) \subseteq$ $T_{1}$ and $T_{1}$ is open.

$T_{1}$ is closed: Let $\left(t^{n}\right)_{n \in \mathbb{N}}$ be a sequence in $T_{1}$ that converges to $t \in T$ with respect to the above defined topology. Suppose for contradiction that $t \in T_{2}$. Let $x=f(t)$. Then $\operatorname{dist}_{G_{f}}\left(a^{*}, x\right)+\operatorname{dist}_{G_{f}}\left(x, a^{*}\right)=\delta+\varepsilon_{x}$ for some $\varepsilon=\varepsilon(x)>0$. Choose $n_{0}$ such that $\sup _{a \in A}\left|t_{a}^{n_{0}}-t_{a}\right|<\varepsilon / 2$. Let $y=f\left(t^{n_{0}}\right)$. As $t^{n_{0}} \in T_{1}$, $\operatorname{dist}_{G_{f}}\left(a^{*}, y\right)+\operatorname{dist}_{G_{f}}\left(y, a^{*}\right)<\delta$.

\footnotetext{
${ }^{5}$ We were inspired by Chung and Olszewski (2007) to use countability this way.
} 
Then the following holds:

$$
\begin{aligned}
\delta+\varepsilon & =\operatorname{dist}_{G_{f}}\left(a^{*}, x\right)+\operatorname{dist}_{G_{f}}\left(x, a^{*}\right) \\
& \leq \operatorname{dist}_{G_{f}}\left(a^{*}, y\right)+\ell_{y x}+\ell_{x y}+\operatorname{dist}_{G_{f}}\left(y, a^{*}\right) \\
& <\delta+\ell_{y x}+\ell_{x y} \\
& \leq \delta+t_{x}-t_{y}+t_{y}^{n_{0}}-t_{x}^{n_{0}} \\
& \leq \delta+\left|t_{x}-t_{x}^{n_{0}}\right|+\left|t_{y}-t_{y}^{n_{0}}\right| \\
& <\delta+\frac{\varepsilon}{2}+\frac{\varepsilon}{2} \\
& =\delta+\varepsilon
\end{aligned}
$$

a contradiction.

Now we abandon the identification of type spaces with vectors in $\mathbb{R}^{A}$ and return to the explicit use of valuation functions. We obtain the following corollary.

Corollary 1 Let $A$ be a countable outcome space. Let each agent $i \in\{1, \ldots, n\}$ have types from the (topologically) connected type space $T_{i} \subseteq \mathbb{R}^{k_{i}}$. Let each agent's valuation function $v_{i}: T_{i} \rightarrow \mathbb{R}^{A}$ be a continuous function with respect to the above defined topology on $\mathbb{R}^{A}$.

Then, every allocation rule $f: \prod_{i=1}^{n} T_{i} \rightarrow A$ that is implementable in dominant strategies satisfies revenue equivalence.

Proof. As the $v_{i}$ are continuous and the $T_{i}$ are connected, $v_{i}\left(T_{i}\right)$ is connected for every agent. The result follows immediately from Theorem 5 .

Notice that continuity of $v_{i}: T_{i} \rightarrow \mathbb{R}^{A}$ with respect to the above defined topology is a different notion than continuity of $v_{i}(a, \cdot)$ for every $a \in A$. Especially, the latter does not imply the former.

An example in Holmström (1979) demonstrates that Theorem 4 and Corollary 1 cannot be generalized to the case of an uncountable outcome space. In that example, there is one agent with a one-dimensional type from a compact interval on the real line and continuous valuation function. The outcome space is a compact interval as well and Holström shows that revenue equivalence fails.

\section{$7 \quad$ Deducing Existing Results}

In this section, we show how the characterization of Chung and Olszewski (2007) follows from our main result and comment briefly on the results in other papers on revenue 
equivalence. First, we introduce the notation used by Chung and Olszewski and restate their characterization theorem.

Let $A$ be finite. Let $B_{1}, B_{2}$ be disjoint subsets of $A$ and $r: B_{1} \cup B_{2} \rightarrow \mathbb{R}$. For every $\varepsilon>0$, let

$$
V_{1}(\varepsilon)=\cup_{b \in B_{1}}\left\{t \in T \mid \forall a \in B_{2}: v(b, t)-v(a, t)>r(b)-r(a)+\varepsilon\right\}
$$

and

$$
V_{2}(\varepsilon)=\cup_{a \in B_{2}}\left\{t \in T \mid \forall b \in B_{1}: v(b, t)-v(a, t)<r(b)-r(a)-\varepsilon\right\} .
$$

Finally, $V_{i}=\cup_{\varepsilon>0} V_{i}(\varepsilon)$. Observe that $V_{1} \cap V_{2}=\emptyset$. Call the type space $T$ splittable if there are $B_{1}, B_{2}$ and $r$ such that $T$ is a subset of $V_{1} \cup V_{2}$ where $V_{i} \neq \emptyset$ for $i=1,2$.

Theorem 6 (Chung and Olszewski 2007) Let $A$ be finite. Then, the following two statements are equivalent.

1. All $f$ that are implementable in dominant strategies satisfy revenue equivalence.

2. $T$ is not splittable.

Notice that in Theorem 3 no assumption on the cardinality of $A$ is made, whereas in Theorem 6, $A$ is assumed finite. On the other hand, Theorem 3 imposes a condition on the allocation rule, whereas Theorem 6 characterizes $T$ and $v$ such that all allocation rules that are implementable in dominant strategies satisfy revenue equivalence. When $A$ is not finite but countable it is shown by Chung and Olszewski that item 2 of Theorem 6 implies revenue equivalence.

In order to show that Statement 2 in Theorem 6 is a necessary condition for revenue equivalence, one can directly construct an allocation rule and two payment schemes that do not differ by a constant from the assumption that $T$ is splittable. This is done in the paper by Chung and Olszewski. We give an alternative proof for the fact that Statement 2 is a sufficient condition. Our proof establishes the connection to the allocation graph defined in Section 4 and assigns an interpretation to the function $r$ defined above.

Proof. $[2 \Rightarrow 1]$ Let $f$ be an allocation rule that is implementable in dominant strategies but does not satisfy revenue equivalence. Since $f$ is implementable in dominant strategies, the allocation graph $G_{f}$ satisfies the non-negative cycle property. Using Theorem 3 , there are $a, b \in A$ such that $\operatorname{dist}_{G_{f}}(a, b)+\operatorname{dist}_{G_{f}}(b, a)>0$. We show that this implies that $T$ is splittable. That is, there exist $B_{1}, B_{2}$, and $r: B_{1} \cup B_{2} \rightarrow \mathbb{R}$ such that there is an $\varepsilon>0$ such that $V_{1}(\varepsilon), V_{2}(\varepsilon) \neq \emptyset$ and $T \subseteq V_{1}(\varepsilon) \cup V_{2}(\varepsilon)$. 
Let

$$
\begin{aligned}
& B_{1}=\left\{c \in A \mid \operatorname{dist}_{G_{f}}(a, c)=-\operatorname{dist}_{G_{f}}(c, a)\right\} \text { and } \\
& B_{2}=\left\{c \in A \mid \operatorname{dist}_{G_{f}}(a, c)>-\operatorname{dist}_{G_{f}}(c, a)\right\} .
\end{aligned}
$$

Clearly, $\left(B_{1}, B_{2}\right)$ is a partition of $A$ and both sets are non-empty. From the definition of $B_{2}$ we get for every $b_{1} \in B_{1}, b_{2} \in B_{2}$ that

$$
\operatorname{dist}_{G_{f}}\left(a, b_{2}\right)+\ell_{b_{2} b_{1}}+\operatorname{dist}_{G_{f}}\left(b_{1}, a\right)>0
$$

since otherwise $a$ and $b_{2}$ would lie on the same 0-length cycle implying $\operatorname{dist}_{G_{f}}\left(a, b_{2}\right)+$ $\operatorname{dist}_{G_{f}}\left(b_{2}, a\right)=0$. As $b_{1} \in B_{1}$, this is equivalent to

$$
\ell_{b_{2} b_{1}}>\operatorname{dist}_{G_{f}}\left(a, b_{1}\right)-\operatorname{dist}_{G_{f}}\left(a, b_{2}\right) \text {. }
$$

As $B_{1}$ and $B_{2}$ are finite, there exists $\delta>0$ such that for all $b_{1} \in B_{1}, b_{2} \in B_{2}$

$$
\ell_{b_{2} b_{1}}>\operatorname{dist}_{G_{f}}\left(a, b_{1}\right)-\operatorname{dist}_{G_{f}}\left(a, b_{2}\right)+\delta \text {. }
$$

Let us define the function $r$ by

$$
r(c)= \begin{cases}\operatorname{dist}_{G_{f}}(a, c)+\delta, & \text { if } c \in B_{1} \\ \operatorname{dist}_{G_{f}}(a, c), & \text { if } c \in B_{2}\end{cases}
$$

Then we get from (1):

$$
\ell_{b_{2} b_{1}}>r\left(b_{1}\right)-r\left(b_{2}\right)
$$

Similarly, from the definition of the dist-function, we get for all $b_{1} \in B_{1}, b_{2} \in B_{2}$

$$
\operatorname{dist}_{G_{f}}\left(a, b_{1}\right)+\ell_{b_{1} b_{2}} \geq \operatorname{dist}_{G_{f}}\left(a, b_{2}\right)
$$

and consequently

$$
\ell_{b_{1} b_{2}}>r\left(b_{2}\right)-r\left(b_{1}\right) .
$$

Due to the finiteness of $B_{1}$ and $B_{2}$ there exists an $\varepsilon>0$ such that for all $b_{1} \in B_{1}, b_{2} \in B_{2}$,

$$
\begin{aligned}
& \ell_{b_{2} b_{1}}>r\left(b_{1}\right)-r\left(b_{2}\right)+\varepsilon, \quad \text { and } \\
& \ell_{b_{1} b_{2}}>r\left(b_{2}\right)-r\left(b_{1}\right)+\varepsilon .
\end{aligned}
$$


Observing that for $t \in f^{-1}\left(b_{1}\right), v\left(b_{1}, t\right)-v\left(b_{2}, t\right) \geq \ell_{b_{2} b_{1}}$ and for $t \in f^{-1}\left(b_{2}\right), v\left(b_{2}, t\right)-$ $v\left(b_{1}, t\right) \geq \ell_{b_{1} b_{2}}$, inequalities (4) and (5) imply that $f^{-1}\left(B_{1}\right) \subseteq V_{1}(\varepsilon)$ and $f^{-1}\left(B_{2}\right) \subseteq V_{2}(\varepsilon)$. Hence, $T \subseteq V_{1}(\varepsilon) \cup V_{2}(\varepsilon)$. Finally, $V_{1}(\varepsilon)$ and $V_{2}(\varepsilon)$ are non-empty, as $B_{1}$ and $B_{2}$ are non-empty, thus $T$ is splittable.

To illustrate the difference between our characterization and that by Chung and Olszewski, we give an example where their characterization cannot be applied, but where Theorem 3 implies revenue equivalence.

Example 4 Reconsider Example 2 from Section 4, where Theorem 3 implied revenue equivalence. If the characterization by Chung and Olszewski applied as well, then all allocation rules implementable in dominant strategies would have to satisfy revenue equivalence for the given type space and valuation functions. To see that this is not the case, consider allocation rule $g$ such that $g(x)=c$ and $g(y)=g(z)=b$. The allocation graph has nodes $b$ and $c$ and arc lengths $\ell_{b c}=v(x, c)-v(x, b)=-1$ and $\ell_{c b}=\inf \{v(y, b)-v(y, c), v(z, b)-v(z, c)\}=2$, which gives a cycle of strictly positive length. Therefore, $g$ is implementable in dominant strategies, but does not satisfy revenue equivalence according to Theorem 3.

Using Theorem 6, Chung and Olszewski derive sufficient conditions for revenue equivalence for the case of countable outcome spaces and for outcome spaces that are the set of all probability distributions over a finite set. For countable outcome spaces, their results coincide with our Theorem 4 and Corollary 1. The results of prior work mentioned in the introduction (Green and Laffont 1977, Holmström 1979, Krishna and Maenner 2001, Milgrom and Segal 2002) follow in the same way from Theorem 4 and Corollary 1 as described by Chung and Olszewski. We therefore refer to their paper for a detailed discussion of the mentioned literature. Furthermore, the sufficient condition that Suijs (1996) derives from his characterization follows easily from our results and the fact that path-connectedness implies connectedness.

\section{Other Notions of Incentive Compatibility}

Our results can be extended to other notions of incentive compatibility. We briefly discuss two of them.

Ex-post incentive compatibility with externalities. The notation used throughout this paper can be used to model allocational externalities, as allocations to all agents can be described by an outcome $a \in A$. In order to account for informational externalities 
in the case of ex-post incentive compatibility, the valuation function of agent $i$ is also a function of the true types of the other agents. An ex-post equilibrium is a Nashequilibrium where every agent knows the types of all other agents. Formally, truth-telling is an ex-post equilibrium for allocation rule $f$ and payment scheme $\pi$ if for all agents $i$, all types $t_{i}$ and $s_{i}$ of $i$ and all types $t_{-i}$ of the other agents

$$
v_{i}\left(f\left(t_{i}, t_{-i}\right), t_{i}, t_{-i}\right)-\pi\left(t_{i}, t_{-i}\right) \geq v_{i}\left(f\left(s_{i}, t_{-i}\right), t_{i}, t_{-i}\right)-\pi\left(s_{i}, t_{-i}\right) .
$$

Again, incentive compatible payment schemes can be associated with node potentials in the allocation graphs the edge lengths in $G_{f}$ have to be defined as follows:

$$
\ell_{a b}=\inf _{t_{i} \in f^{-1}(b)}\left(v_{i}\left(b, t_{i}, t_{-i}\right)-v_{i}\left(a, t_{i}, t_{-i}\right)\right)
$$

We get the following result.

Theorem 7 An ex-post implementable allocation rule $f$ satisfies revenue equivalence if and only if in every allocation graph $G_{f}$, for every two allocations $a, b$, $\operatorname{dist}_{G_{f}}(a, b)+$ $\operatorname{dist}_{G_{f}}(b, a)=0$.

Bayes-Nash incentive compatibility. Müller, Perea, and Wolf (2007) show how to construct a graph similar to the allocation graph for the case of Bayes-Nash incentive compatibile allocation rules. In this setting, the (multi-dimensional) types of all agents are independently distributed random vectors. The notation used by Müller, Perea, and Wolf accounts for allocational as well as informational externalities, that is, the valuation of an agent depends on the chosen allocation, the type of the agent and the types of all other agents. An allocation rule $f$ is said to be Bayes-Nash incentive compatible if there is a payment scheme $\pi$ such that for all agents $i$ and all types $t_{i}$ and $s_{i}$ of $i$ the following is true

$$
E_{-i}\left[v_{i}\left(f\left(t_{i}, t_{-i}\right), t_{i}, t_{-i}\right)-\pi_{i}\left(t_{i}, t_{-i}\right)\right] \geq E_{-i}\left[v_{i}\left(f\left(s_{i}, t_{-i}\right), t_{i}, t_{-i}\right)-\pi_{i}\left(s_{i}, t_{-i}\right)\right]
$$

where the expected value is taken with respect to the types of all agents other than $i$. That is, truth-telling of every agent is a Nash equilibrium when agents try to maximize expected utilities. For every agent $i$, one can construct a single complete directed graph, the type graph $T_{f}^{i}$, where there is a node for each type of the agent and the length of the edge from type $s_{i}$ to type $t_{i}$ is defined as follows:

$$
\ell_{s_{i} t_{i}}=E_{-i}\left[v_{i}\left(f\left(t_{i}, t_{-i}\right), t_{i}, t_{-i}\right)-v_{i}\left(f\left(s_{i}, t_{-i}\right), t_{i}, t_{-i}\right)\right] .
$$


Incentive compatible payment schemes can then be associated with node potentials in the type graph.

Theorem 8 A Bayes-Nash implementable allocation rule $f$ satisfies revenue equivalence if and only if in the type graph $T_{f}^{i}$ of $f$ with respect to any agent $i$, for all types $s_{i}$ and $t_{i}$ of this agent, dist $t_{T_{f}}\left(s_{i}, t_{i}\right)+$ dist $_{T_{f}}\left(t_{i}, s_{i}\right)=0$.

\section{References}

Chung, K.-S. and W. Olszewski (April 2007). A non-differentiable approach to revenue equivalence. Retrieved from http://facultyweb.at.northwestern.edu/economics/chung/.

Green, J. and J.-J. Laffont (1977). Characterization of satisfactory mechanisms for the revelation of preferences for public goods. Econometrica 45(2), 427-438.

Gui, H., R. Müller, and R. Vohra (2004, October). Dominant strategy mechanisms with multidimensional types. Discussion Paper 1392, The Center for Mathematical Studies in Economics \& Management Sciences, Northwestern University, Evanston, IL.

Holmström, B. (1979). Groves' scheme on restricted domains. Econometrica 47(5), $1137-1144$.

Klemperer, P. (1999). Auction theory: A guide to the literature. Journal of Economic Surveys 13(3), 227-286.

Krishna, V. and E. Maenner (2001). Convex potentials with an application to mechanism design. Econometrica 69(4), 1113-1119.

Milgrom, P. and I. Segal (2002). Envelope theorems for arbitrary choice sets. Econometrica $70(2), 583-601$.

Müller, R., A. Perea, and S. Wolf (2007). Weak monotonicity and Bayes-Nash incentive compatibility. Games and Economic Behavior. To appear. Available as METEOR research memorandum $\mathrm{RM} / 05 / 039$.

Munkres, J. R. (2000). Topology (2nd ed.). Prentice Hall.

Myerson, R. (1981). Optimal auction design. Mathematics of Operations Research 6(1), $58-73$.

Rochet, J.-C. (1987). A necessary and sufficient condition for rationalizability in a quasi-linear context. Journal of Mathematical Economics 16(2), 191-200. 
Saks, M. and L. Yu (2005). Weak monotonicity suffices for truthfulness on convex domains. In Proc. 6th ACM conference on Electronic Commerce, pp. 286 - 293.

Suijs, J. (1996). On incentive compatibility and budget balancedness in public decision making. Economic Design 2, 193-209. 